\title{
An Improved Algorithm of Narrow-Band Interference Rejection Based on Time-Domain Adaptive Filtering in DSSS System Rui-Jun WANG ${ }^{1}$, Shi-Guang $\mathrm{HAO}^{1,2}$, Shu-Yun $\mathrm{LI}^{1}$, Sheng $\mathrm{KE}^{1{ }^{*}}$ and
Shuai WANG
}

${ }^{1}$ School of Information and Electronics, Beijing Institute of Technology, China

${ }^{2}$ Department of Telecommunication Satellite, Academy of Space Technology, China

Keywords: Direct sequence spread spectrum, Narrow-Band interference, Timedomain adaptive filter, Least-mean-square algorithm

\begin{abstract}
In Direct sequence spread spectrum (DSSS) communication system, the auto-correlation of Pseudo-Noise Code is weak while the auto-correlation of Narrow-Band Interference (NBI) is strong, so that the NBI is more predictable than the DSSS signal. The principle of time-domain anti-interference is the auto-correlation difference between DSSS signal and NBI. However, due to the existence of oversampling in the transmitter, the auto-correlation of DSSS signal increases significantly, and the difference of auto-correlation between DSSS signal and NBI will diminish. And then the traditional time-domain adaptive filter will introduce distortion to the DSSS signal in rejection of the NBI. In this paper, an improved time-domain adaptive filtering algorithm is proposed. This algorithm is based on the interpolating transversal filter and the Least-mean-square (LMS) algorithm for updating the weights adaptively. Its anti-interference ability gains about $10 \mathrm{~dB}$ than the traditional adaptive filter under the situation of SNR/chip is greater than 50dB. Both theoretical analysis and simulations illustrate that the new algorithm has a stronger anti-interference ability and introduces less distortion to the signal under high SNR conditions. Meanwhile, it has similar performance with the traditional adaptive filter under low SNR conditions.
\end{abstract}

\section{Introduction}

Direct Sequence Spread Spectrum (DSSS) has been widely employed in the field of military and civilian communication, for the advantages of anti-interference, low probability of interception, high security, and Code Division Multiple Access (CDMA) communication[1]. Narrow-Band Interference (NBI) is the common interference pattern in the field of military confrontation, and the power spectrum density of NBI can reach very high. Sometimes the NBI can result in a sharp decline of the performance in DSSS system. DSSS itself has a certain amount of anti-interference ability, which is determined by the spreading gain. However, when the power of the interference existed in the channel exceeds the spreading gain of the DSSS, the performance of the DSSS system will be seriously deteriorated. Under such circumstances, anti-interference measures must be adopted at the receiving end before the de-spreading process [2].

The common anti-interference methods can be roughly divided into four fields, which are the antenna technique, the time-domain anti-interference, the transform-domain anti-interference, and the code-aided technique. Among these methods, the time-domain anti-interference method has been widely used due to its advantages such as good interference suppression performance, less resource 
consumption, less sampling points needed, low algorithm complexity, easy realizationof hardware and so on[3,4]. The time-domain anti-interference mainly refers to the interference estimation and offset. Given $\mathrm{n}$ points $y_{n}, y_{n-1} \ldots y_{2}, y_{1}$ in the observation space, use the $\mathrm{n}$ points to estimate the interference existed in $y_{i}$ and eliminate it. If " $1 \leq i \leq n$ ", this structure is called "Prediction filter". Else if " $i>n$ or $\mathrm{i}<1$ ", this structure is called "Interpolation filter ". Our discussion as follows is mainly based on the second kind of filter.

Many papers had talked about the adaptive filter, such as [5], but there seems to be practically no studies were talking under oversampling conditions. And we will launch our research under such conditions. This paper first studies the traditional time-domain adaptive filtering narrow-band interference suppression algorithm, and then analyzes the deficiency of the traditional algorithm through a simulation. Second, this paper proposes an improved algorithm based on time-domain anti-interference and LMS algorithm, which we call " Interval adaptive filtering algorithm". Through detailed theoretical derivation and simulation, this paper proves that the new algorithm has a stronger interference suppression ability, which is about $10 \mathrm{~dB}$ higher than the traditional adaptive filter under the situation of SNR/chip is greater than $50 \mathrm{~dB}$ and the NBI bandwidth is 10 percent of the DSSS signal. While under low SNR conditions, the performances of the two algorithms are similar to each other.

\section{Analysis of The Traditional Adaptive Filter}

The principle of the time-domain anti-interference method is mainly based on the difference between DSSS signal and NBI in auto-correlation degree. Auto-correlation of the DSSS signal would be weak when the sampling rate is equal to the chip rate, so that we can't estimate the current sampling value from the past sampling point. While, auto-correlation of the NBI can be rather strong, so that estimation of the interference would be easy to obtain from the past sampling point. Subtracting the estimated interference from the current sample value, the output is the desired DSSS signal[6].

However, in [7], when the sampling rate in the receiver is higher than the chip rate, auto-correlation of the DSSS signal will increase significantly, leading to that a part of the signal is also estimated when predicting the NBI, which will cause damage to the signal and limit the system performance.

\section{A. System Structure}

As is shown in Fig. $1, x_{i+N}, \ldots, x_{i+1}, x_{i}, x_{i-1}, x_{i-N}$ are base-band data. $x_{i}$ is the sampling value to be estimated. Tis the sampling interval. $\alpha_{-\mathrm{N}}, \ldots, \alpha_{-1}, \alpha_{1}, \ldots, \alpha_{\mathrm{N}}$ are tap coefficients of the filter. The coefficients will change themselves adaptively according to the input data, and eventually converge to Wiener solution. $e_{i}$ is the output of the desired signal after filtering, which does not contain the interference component but only the signal component in the ideal situation [8]. 


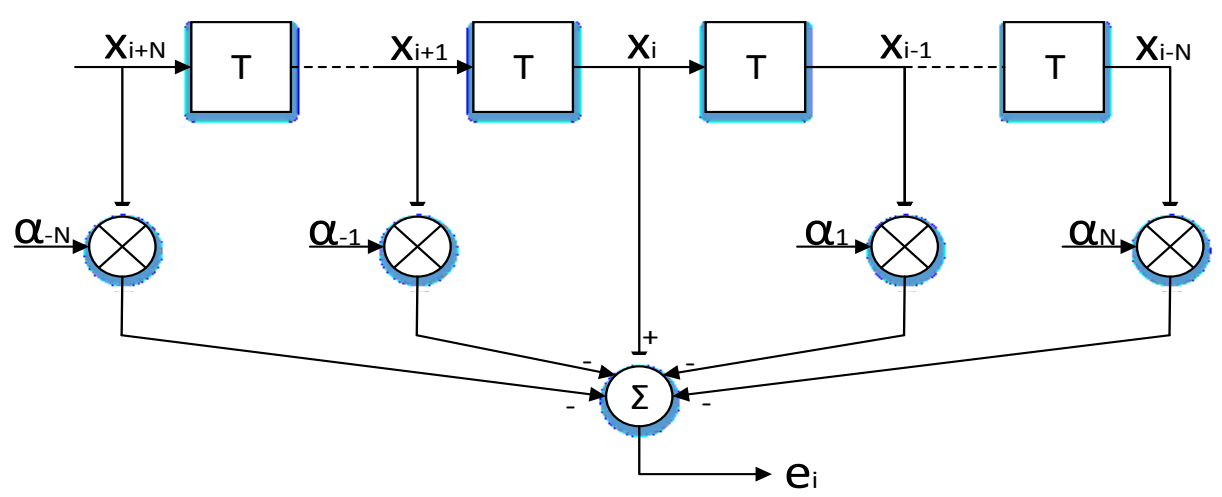

Figure.1 Structure of the traditional adaptive filter

\section{B. Performance Analysis}

First of all, we assume that the DSSS signal, the NBI, and the additive Gauss white noise are uncorrelated to each other. And then we analyze the performance of the traditional adaptive filter under high SNR conditions without interference.

The input signal vector is :

$$
\boldsymbol{X}_{\boldsymbol{i}}=\left[x_{i+N}, \ldots, x_{i+1}, x_{i-1}, \ldots, x_{i-\mathrm{N}}\right]^{T}
$$

The tap coefficients vector is:

$$
\boldsymbol{w}=\left[\alpha_{-\mathrm{N}}, \ldots, \alpha_{-1}, \alpha_{1}, \ldots, \alpha_{\mathrm{N}}\right]^{T}
$$

The desired signal is:

$$
e_{i}=x_{i}-\boldsymbol{w}^{H} \boldsymbol{X}_{\boldsymbol{i}}
$$

When the step length factor is well selected, the tap coefficients will converge to the Wiener solution $\boldsymbol{w}_{\text {opt }}$, which satisfies the Wiener-Hof $(\mathrm{W}-\mathrm{H})$ equation:

$$
\boldsymbol{R}_{x x} \boldsymbol{w}_{\text {opt }}=\boldsymbol{r}_{x d}
$$

Where $\boldsymbol{R}_{\boldsymbol{x} x}$ represents the auto-correlation matrix of the input signal vector, and $\boldsymbol{r}_{\boldsymbol{x} \boldsymbol{d}}$ represents the cross-correlation vector of the input signal vector and the desired signal. $\boldsymbol{R}_{\boldsymbol{x}}=\mathrm{E}\left[\boldsymbol{X}_{\boldsymbol{i}} \boldsymbol{X}_{\boldsymbol{i}}^{\boldsymbol{H}}\right]$ and $\boldsymbol{r}_{\boldsymbol{x} \boldsymbol{d}}=\mathrm{E}\left[x_{i} \boldsymbol{X}_{\boldsymbol{i}}\right]$. Substitute $\boldsymbol{X}_{\boldsymbol{i}}$ into these two equations, then we will get the expansion expression of $\boldsymbol{R}_{\boldsymbol{x} x}$ and $\boldsymbol{r}_{\boldsymbol{x} \boldsymbol{d}}$.

$$
\boldsymbol{R}_{x x}=\left[\begin{array}{ccccccc}
r_{x x}(0) & r_{x x}(-1) & \ldots & r_{x x}(-N+1) & r_{x x}(-N-1) & \ldots & r_{x x}(-2 N) \\
r_{x x}(1) & r_{x x}(0) & \ldots & r_{x x}(-N+2) & r_{x x}(-N) & \ldots & r_{x x}(-2 N+1) \\
\vdots & \vdots & \vdots & \vdots & \vdots & \vdots & \vdots \\
r_{x x}(N-1) & r_{x x}(N-2) & \ldots & r_{x x}(0) & r_{x x}(-2) & \ldots & r_{x x}(-N-1) \\
r_{x x}(N+1) & r_{x x}(N) & \ldots & r_{x x}(2) & r_{x x}(0) & \ldots & r_{x x}(-N+1) \\
\vdots & \vdots & \vdots & \vdots & \vdots & \vdots & \vdots \\
r_{x x}(2 N) & r_{x x}(2 N-1) & \ldots & r_{x x}(N+1) & r_{x x}(N-1) & \ldots & r_{x x}(0)
\end{array}\right]_{2 N * 2 N}
$$

$\boldsymbol{r}_{\boldsymbol{x} \boldsymbol{d}}=\left[r_{x x}(-\mathrm{N}) \ldots r_{x x}(-1) r_{x x}(1) \ldots r_{x x}(\mathrm{~N})\right]$

Where $r_{x x}(\mathrm{n})$ is the auto-correlation function of the input signal. Under high SNR conditions without interference, the input signal vector $\boldsymbol{X}_{\boldsymbol{i}}$ can be viewed as two times oversampled DSSS signal. And $r_{x x}$ (n)equals to 0 unless $n$ equals to 0,1 , or -1 , which is: 


$$
r_{x x}(\mathrm{n})=\left\{\begin{array}{cc}
r_{0} & n=0 \\
r_{1} & n=1 \\
r_{1}{ }^{*} & n=-1 \\
0 & \text { others }
\end{array}\right\}
$$

Substitute (7) into (5) and (6), and then we will get the simplified expression of $\boldsymbol{R}_{\boldsymbol{x} x}$ and $\boldsymbol{r}_{\boldsymbol{x} \boldsymbol{d}}$.

$$
R_{x x}=\left[\begin{array}{ll}
A & 0 \\
0 & A
\end{array}\right]
$$

Where

$$
\boldsymbol{A}=\left[\begin{array}{cccccc}
r_{0} & r_{1}{ }^{*} & 0 & 0 & \ldots & 0 \\
r_{1} & r_{0} & r_{1}{ }^{*} & 0 & \ddots & \vdots \\
0 & r_{1} & r_{0} & \ddots & \ddots & 0 \\
0 & 0 & \ddots & \ddots & r_{1}{ }^{*} & 0 \\
\vdots & \ddots & \ddots & r_{1} & r_{0} & r_{1}{ }^{*} \\
0 & \ldots & 0 & 0 & r_{1} & r_{0}
\end{array}\right]_{N * \mathrm{~N}}
$$

$\boldsymbol{r}_{\boldsymbol{x} \boldsymbol{d}}=\left[\begin{array}{lllllllll}0 & 0 & \ldots & 0 & r_{1}{ }^{*} r_{1} & 0 & \ldots & 0 & 0\end{array}\right]^{T}$

According to (4), we can obtain the two tap coefficients in the middle of the filter:

$$
\begin{aligned}
& \alpha_{-1}=\frac{r_{0} r_{1}{ }^{*}}{r_{0}^{2}-\left|r_{1}\right|^{2}} \\
& \alpha_{1}=\frac{r_{0} r_{1}}{r_{0}^{2}-\left|r_{1}\right|^{2}}
\end{aligned}
$$

And then the mean square power of the desired signal is:

$$
\begin{gathered}
\mathrm{E}\left[\left|e_{i}\right|^{2}\right]=\mathrm{E}\left[\left|x_{i}\right|^{2}\right]-\boldsymbol{w}_{\boldsymbol{o p t}}{ }^{\boldsymbol{H}} \boldsymbol{r}_{\boldsymbol{x} \boldsymbol{d}} \\
=\mathrm{r}_{0}-\frac{2 \mathrm{r}_{0}\left|\mathrm{r}_{1}\right|^{2}}{\mathrm{r}_{0}{ }^{2}-\left|\mathrm{r}_{1}\right|^{2}}
\end{gathered}
$$

According to the ideal auto-correlation function of DSSS signal, we have the equation $\left|\mathbf{r}_{\mathbf{1}}\right| \approx 0.5 \mathbf{r}_{\mathbf{0}}$. Substitute this equation into (12) and then we will get:

$\mathrm{E}\left[\left|e_{i}\right|^{2}\right]=0.33 r_{0}$

From (13) we can see that, under high SNR conditions without interference, about $5 \mathrm{~dB}$ attenuation will be introduced to the DSSS signal by traditional adaptive filter [8].

\section{Simulation}

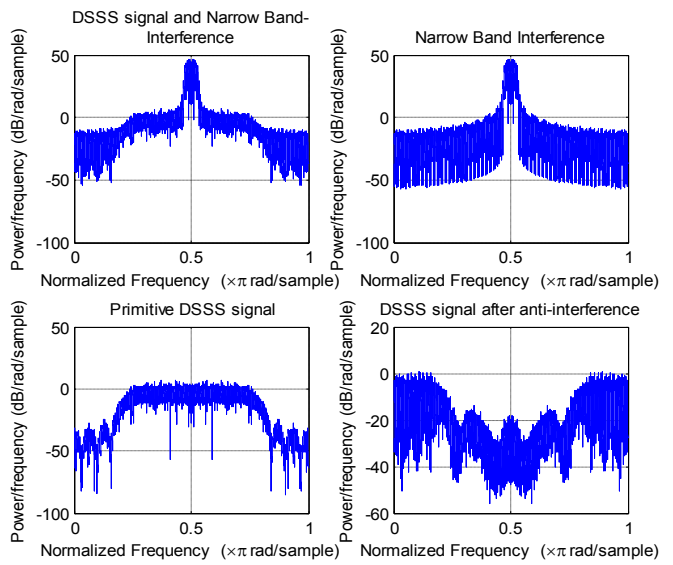

Figure. 2 Simulation of the traditional adaptive filter performance 
Fig. 2 is the power spectrum simulation of the DSSS signal. The simulation conditions are as follows: Spread ratio is 127; Oversampling ratio is $4 ; \boldsymbol{E}_{\boldsymbol{b}} / \boldsymbol{N}_{\mathbf{0}}$ is $70 \mathrm{~dB}\left(\boldsymbol{E}_{\boldsymbol{b}} / \boldsymbol{N}_{\mathbf{0}}\right.$ is $\mathrm{SNR} /$ chip); Interference bandwidth is 10 percent of the DSSS signal bandwidth; Interference-signal power ratio (ISR) is $30 \mathrm{~dB}$; The order of the filter is 30 .

The third figure in Fig.2 is the power spectrum of the primitive DSSS signal before filtering, and the fourth figure is the power spectrum of the DSSS signal after filtering. From the contrast of these two figures we can see that the traditional adaptive filter will introduce about $30 \mathrm{~dB}$ attenuation to the DSSS signal under high SNR conditions, which is consistent with the previous theoretical analysis.

\section{Interval Adaptive Filter}

As is mentioned, the reason why traditional adaptive filter will cause damage to the DSSS signal is that the oversampling increases the auto-correlation of the signal. One method to reduce the damage is to decrease the sampling rate. However, this method also has a serious flaw, which is loss of the useful information $[9,10]$.Therefore, this paper proposes the interval adaptive filter algorithm, which will not cause damage to the signal while suppressing the NBI, and it does not need decreasing the sampling rate in the receiver end.

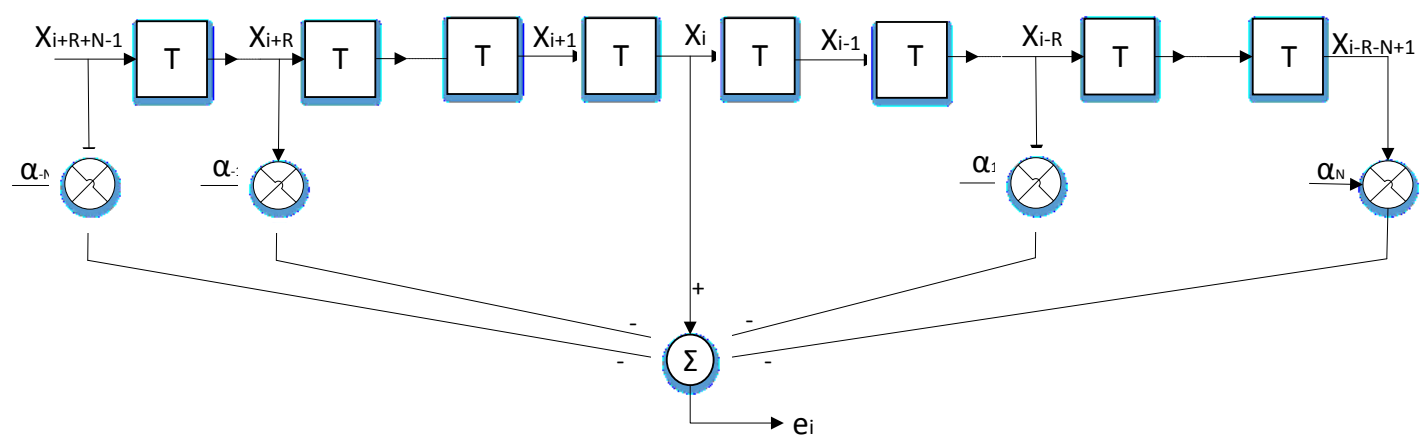

Figure 3. Structure of the interval adaptive filter

Supposing the oversampling rate is $\mathrm{R}$, so that the $\mathrm{R}-1$ points in the left side and the $\mathrm{R}-1$ points in the right side of the estimated point $\boldsymbol{x}_{\boldsymbol{i}}$ are probably related to it. The main principle of the interval adaptive filter is that avoiding using the nearest $2(\mathrm{R}-1)$ points of $\boldsymbol{x}_{\boldsymbol{i}}$ to estimate the interference, and this method will decrease the auto-correlation and thus reduce the damage caused to the DSSS signal.

Fig.3 is the structure of the interval adaptive filter, where $R$ represents the oversampling rate and the rest symbols are same as those in Fig.1. We avoid 2(R-1) points on both sides of the estimated point when predicting the interference, so that the estimated value does not contain the signal components but only the interference components. For convenience, we analyze the performance of the interval filter in the case $\mathrm{R}=2$, and similar results can be obtained when $\mathrm{R}$ takes other values. The mathematical derivation is as follow.

The input signal vector is:

$$
\mathbf{X}_{\mathbf{i}}=\left[\mathrm{X}_{\mathrm{i}+\mathrm{N}+1}, \ldots, \mathrm{X}_{\mathrm{i}+2}, \mathrm{X}_{\mathrm{i}-2}, \ldots, \mathrm{X}_{\mathrm{i}-\mathrm{N}-1}\right]^{\mathrm{T}}
$$

The tap coefficients vector is:

$$
\mathbf{w}=\left[\alpha_{-N}, \ldots, \alpha_{-1}, \alpha_{1}, \ldots, \alpha_{N}\right]^{T}
$$


The desired signal is:

$$
e_{i}=x_{i}-\boldsymbol{w}^{H} \boldsymbol{X}_{\boldsymbol{i}}
$$

According to (4), we have:

$$
w_{o p t}=R_{x x}{ }^{-1} r_{x d}
$$

Where

$$
\begin{aligned}
& \boldsymbol{R}_{x x} \\
& =\left[\begin{array}{ccccccc}
r_{x x}(0) & r_{x x}(-1) & \ldots & r_{x x}(-N+1) & r_{x x}(-N-3) & \ldots & r_{x x}(-2 N-2) \\
r_{x x}(1) & r_{x x}(0) & \ldots & r_{x x}(-N+2) & r_{x x}(-N-2) & \ldots & r_{x x}(-2 N-1) \\
\vdots & \vdots & \vdots & \vdots & \vdots & \vdots & \vdots \\
r_{x x}(N-1) & r_{x x}(N-2) & \ldots & r_{x x}(0) & r_{x x}(-4) & \ldots & r_{x x}(-N-3) \\
r_{x x}(N+3) & r_{x x}(N+2) & \ldots & r_{x x}(4) & r_{x x}(0) & \ldots & r_{x x}(-N+1) \\
\vdots & \vdots & \vdots & \vdots & \vdots & \vdots & \vdots \\
r_{x x}(2 N+2) & r_{x x}(2 N+1) & \ldots & r_{x x}(N+3) & r_{x x}(N-1) & \ldots & r_{x x}(0)
\end{array}\right]_{2 N * 2 N} \\
& \mathbf{r}_{\mathrm{xd}}=\left[\begin{array}{lllll}
\mathrm{r}_{\mathrm{xx}}(-N-1) & \ldots & \mathrm{r}_{\mathrm{xx}}(-2) \mathrm{r}_{\mathrm{xx}}(2) & \ldots & \left.\mathrm{r}_{\mathrm{xx}}(N+1)\right]
\end{array}\right. \\
& \text { According to (17), we will get: } \boldsymbol{w}_{\text {opt }}=\mathbf{0}
\end{aligned}
$$

Now the tap coefficients are all 0 , and the interval adaptive filter is equivalent to an all-pass filter and then:

$$
e_{i}=x_{i} \mathrm{E}\left[\left|e_{i}\right|^{2}\right]=\mathrm{r}_{0}
$$

It is not difficult to see that, under high SNR conditions without interference, the interval adaptive filter nearly causes no damage to the DSSS signal, which makes up the deficiency of the traditional adaptive filter.

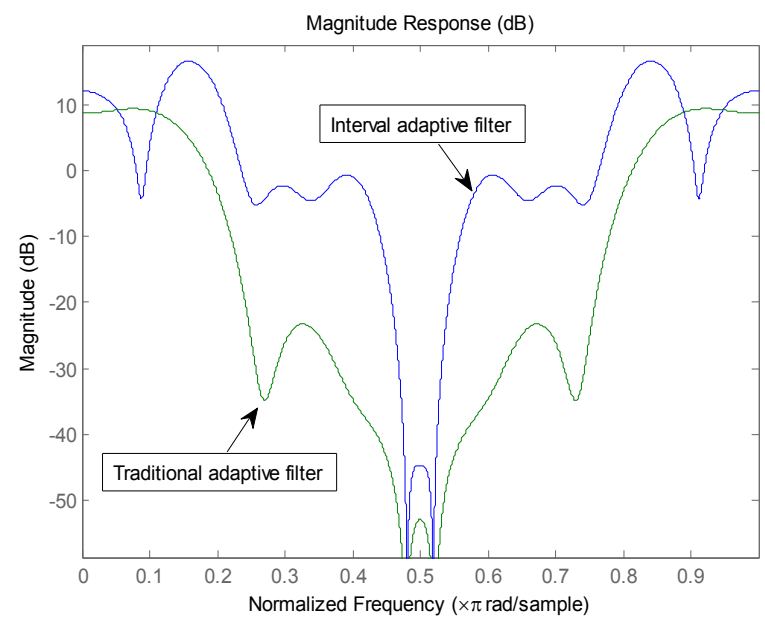

Figure. 4 Amplitude-frequency response of the tap coefficients

Fig. 4 is the amplitude-frequency response of the tap coefficients which have converged to the stationary value. The simulation condition is same as that in Fig.2. As we can see, the degrees of interference suppression of the two kinds of filters are similar. However, the traditional adaptive filter causes $30 \mathrm{~dB}$ attenuation to DSSS signal while the interval adaptive filter nearly does not cause any attenuation. And the flatness of the interval adaptive filter in pass-band is also better than that of the traditional adaptive filter. 


\section{Performance Simulation}

Now we will give the simulation of the performance of the two filters. Without loss of generality, the simulation conditions are as follows: BPSK modulation; Spread ratio is 127; Oversampling ratio is 4; Interference bandwidth is 10 percent of the DSSS signal bandwidth; The order of the filter is 30 double-band.

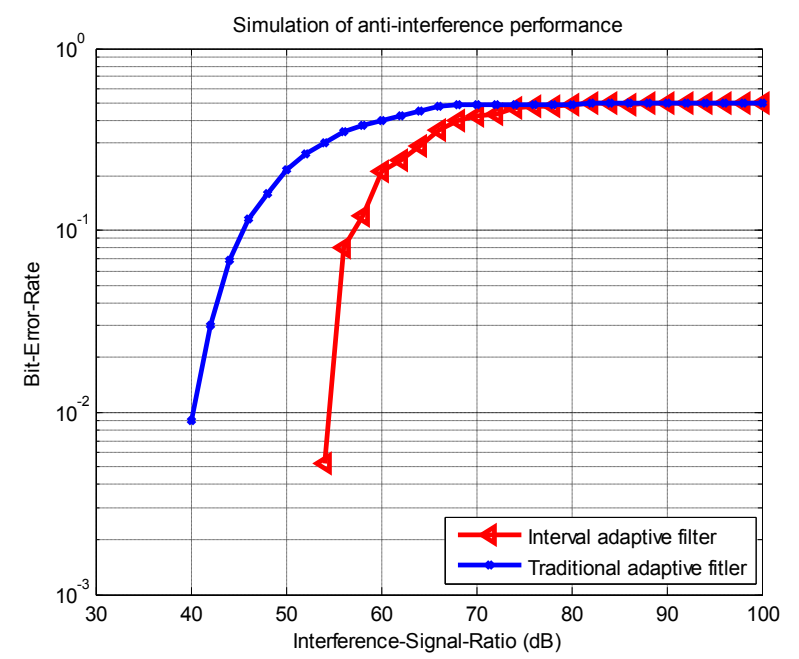

Figure.5 Simulation of anti-interference performance at High SNR

Fig.5 is a simulation of the anti-interference performances of the traditional adaptive filter and the interval adaptive filter under high SNR conditions given $E_{b} / N_{0} 50 \mathrm{~dB}$. The abscissa represents ISR, and the ordinate represents Bit-Error-Rate (BER). With the increase of the ISR, the BER is also increasing. When ISR $<60 \mathrm{~dB}$, the anti-interference performance of the interval adaptive filter is obviously superior to the traditional adaptive filter. The traditional adaptive filter will be completely ineffective when ISR $>$ $64 \mathrm{~dB}$, while the ineffective point of the interval adaptive filter is ISR $>74 \mathrm{~dB}$. We can see that the anti-interference ability of the interval adaptive filter is $10 \mathrm{~dB}$ higher than that of the traditional adaptive filter under high SNR conditions.

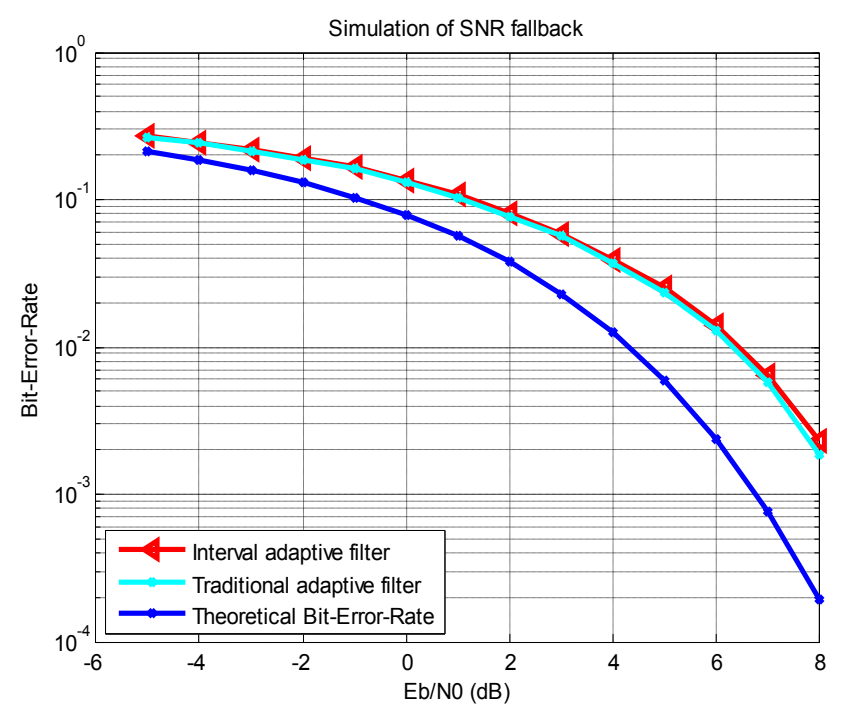

Figure.6 Simulation of SNR fallback

Fig.6 is a simulation of the SNR fallback of the two kinds of filters under the conditions of ISR $=45 \mathrm{~dB}$. The abscissa represents $E_{b} / N_{0}$, and the ordinate represents BER. As we can see, the BER curves of the traditional adaptive filter and the interval 
adaptive filter almost coincide, which indicates that performances of the two kinds of filters are similar under low SNR conditions. The value of SNR fallback compared to the theoretical BER curve is about $2 \mathrm{~dB}$.

\section{Summary}

Based on time-domain anti-interference method, this paper proposes an interval adaptive filtering algorithm, which makes up the deficiency that traditional adaptive filter will cause damage to DSSS signal. Through detailed derivation and simulation, we prove that the new algorithm is better than the traditional filtering algorithm in anti-interference ability under high SNR conditions, and performances of the two algorithms are similar under low SNR conditions. While in aspect of resource consumption, the two algorithms are nearly the same to each other.

Conclusion: the interval adaptive filter is better than the traditional adaptive filter on the whole, and it has a strong practicality.

\section{References}

[1] Cai-jie Xia, Jian-ping An, and Ai-hua Wang, New Technique for Estimating and Suppressing Narrowband Interference in DSSS Systems, Journal of Beijing Institute of Technology, vol. 16, NO.3, November 2007, In Chinese.

[2] Jun-feng Wang,Adaptive Filter for Suppressing Narrow-Band Interference in DSSS Communication, 2011 InternationalConferenceon Computer Science and Services System, vol. 1, pp.162-165, January 2011.

[3] Zhang-feng Wang, Ming Lv and Bin Tang,Paper Application of Partial Coefficient Update LMS Algorithm to Suppress Narrow-Band Interference in DSSS System, 2009 InternationalConferenceon Communication Software and Networks, vol. 1, pp.257-260, January 2009.

[4] Ji-ning Feng, Si-liang Wu, Wei Jiang and Jia-qi Li,Study and Comparison of Filtering Performance to Narrow Band Interference Suppression in DSSS, Transactions of Beijing Institute of Technology, vol. 30, No.6, pp. 727-731, June 2010, In Chinese.

[5] Leslie A.Rusch, and H.Vincent Poor, Narrowband Interferers Suppression in CDMA Spread-Spectrum Communications, IEEE Transactions on Communication, vol. 42, No.2-4, pp. 1969-1979, APRIL 1994.

[6] John W.Ketchum and John G.Proakis, Adaptive Algorithms for Estimating and Suppressing Narrow-Band Interference in PN Spread-Spectrum Systems,IEEE Transactions on Communication, vol. 30, No.5, pp.913-924, May 1982.

[7] Elias Masry,Closed-Form Analytical Results for the Rejection of Narrow-Band Interference in PN Spread-Spectrum System - Part I: Linear Prediction Filters, IEEE Transactions on Communication,vol. 32, NO.8, pp.888-896, August 1984.

[8] Xiao-tian Shao,Research and FPGA realization of Narrow-band Interference Suppression Techniques Based on Time-domain Adaptive Filter,Beijing Institute of Technology, January 2015, In Chinese.

[9]Zhi-guo Sun, Lili Guo and Lin Qi,Narrow-Band Interference Suppression in DSSS Using Adaptive Least-Square Lattice Interpolators, Proceedings of 2006 8th International Conference on Signal Processing, vol. 1, pp.366-368, November 2006. 
[10]Yan-guan Chen, Wen-rui Ding and Chun-hui Liu,Application Research of Frequency Domain Block LMS Algorithm for Anti-jamming in DSSS System, Journal of Telemetry, Tracking and Command, vol. 36, No.3, pp .46-53, May 2015, In Chinese.

[11]Zhao-lin Zhang, Lu Li, Ru-gui Yao and Ling Wang,Narrow-band Interference Detection Technique Based on Least Mean Square Algorithm,Journal of Northwestern Polytechnical University, vol. 34, No.1, pp. 92-97, February 2016.

[12]Xiao-gang Wang and Jie Zhang,LMS Algorithm Suppresses Narrow-band Interference in DSSS, Computer Simulation, vol. 28, No.5, May 2011, In Chinese.

[13] Li-li Guo, Fu-lian Yin and Man-hong Lu, Overview on NBI Suppression of DSSS CDMA Systems, ACTA Electronica Sinica, vol. 37, No.10, October 2009, In Chinese.

[14] Jiang-zhou Wang and Laurence B.Milstein, Adaptive LMS filters for cellular CDMA overlay situations, IEEE Journal on Selected Area in Communications, vol. 14, No.8, pp. 1548-1559, OCTOBER 1996.

[15] Wei Zhang and Tian-ren Yao, Narrow-band Interference Rejection Techniques in DSSS, Communications Technology, No.9, pp.24-27, 2002, In Chinese. 